\title{
Técnicas instrumentales para la evaluación del envejecimiento en los asfaltos
}

\author{
Juliana Puello Méndez \\ Ingeniera Química, M.Sc. Candidata Ph.D., \\ Universidad Industrial de Santander \\ Docente Facultad Ingeniería Industrial e Ingeniería Mecatrónica, \\ Universidad Santo Tomás de Aquino \\ Bucaramanga, Colombia \\ julianapuello@hotmail.com
}

\author{
Natalia Nikolaevna Afanasjeva \\ Química, M.Sc., Ph.D., Instituto del Gas y Petróleo de Moscú \\ Profesora Titular, Departamento de Química, \\ Universidad del Valle \\ Cali, Colombia \\ nafanasjeva@univalle.edu.co
}

\begin{abstract}
Resumen- Desde hace varias décadas, la disminución de yacimientos de crudos livianos en el mundo ha generado la necesidad de explotar yacimientos de crudos pesados, hecho que trae consigo un sinnúmero de desafíos para el aprovechamiento de estos recursos no convencionales. Considerando que la mayor proporción de corrientes derivadas de los crudos pesados la constituyen las fracciones pesadas (asfaltos, fuel oil), se hace relevante el análisis de las propiedades físicas y químicas de estos derivados, con el fin de aportar al desarrollo de tecnologías para el óptimo procesamiento de este tipo de fuentes de combustibles fósiles.
\end{abstract}

En esta investigación se estudian dos asfaltos industriales colombianos y un asfalto venezolano de referencia, mediante técnicas instrumentales, con el fin de comparar parámetros composicionales, funcionales y estructurales, desde el punto de vista del proceso de envejecimiento termo-oxidativo acelerado.

Las técnicas usadas fueron: separación asfaltenos-maltenos por precipitación y filtración, cromatografía líquida en columna empacada para el fraccionamiento de maltenos, espectrometría de infrarrojo con transformada de Fourier (FTIR), y osmometría de presión de vapor (VPO).

Los resultados obtenidos comprenden los cambios con el envejecimiento en la composición fraccional de los asfaltos, en el peso molecular de los asfaltos y sus respectivas fracciones de asfaltenos y maltenos, y en los parámetros funcionales y estructurales obtenidos mediante FTIR.

Con base en los resultados se concluye que es posible caracterizar los asfaltos mediante las técnicas instrumentales, puesto que permiten detectar cambios en la composición y estructura de los mismos, y a la vez los cambios están relacionados con el comportamiento macroscópico de los materiales bituminosos.

Palabras clave-Asfaltos, asfaltenos, maltenos, Envejecimiento acelerado, VPO, FTIR, Cromatografía líquida.

Abstract-During the last decades, reserves of light crude oils have been decreasing, generating a number of cha- llenges related to the production and processing of non conventional sources, such as heavy crude oils. Considering the high content of heavy fractions derived from heavy crude oils, it is necessary to carry on the studies that give a deep insight about physical and chemical properties of bituminous materials, so technologies can be developed for processing crude oils in a proper way.

In this work, two industrial asphalts produced in Colombia, and an asphalt produced in Venezuela are characterized by instrumental techniques, considering the changes that take place during accelerated thermo-oxidative aging.

The techniques employed were: Asphaltenes-maltenes separation by precipitation and filtration, liquid cromathography in a packed column for maltenes fractionation, Fourier-Transform Infrared Spectroscopy (FTIR) and Vapor Pressure Osmometry (VPO).

The results were the changes in fractional composition of asphalts, their molecular weights (asphalts, asphaltenes and maltenes) and the functional and structural parameters obtained by FTIR.

It was found that it is possible to characterize asphalt binders based on instrumental techniques, since these let identify compositional and structural changes, which are related to the macroscopic behavior of bituminous materials.

Keywords-Asphalts, asphaltenes, maltenes, accelerated aging, VPO, FTIR, Liquid chromatography.

\section{INTRODUCCIÓN}

Los asfaltos son sistemas que poseen un balance muy delicado entre compuestos polares y nopolares, de tipo aromático o parafínico, y algunos metales. Esta mezcla de delicados balances juega un importante papel en el envejecimiento y las propiedades de desempeño de los asfaltos [1]. Cualquier desbalance en la química de esta 
mezcla resulta en incompatibilidad, así como menor desempeño y durabilidad del asfalto, debido a que un tipo de compuesto domina la mezcla a expensas de otro grupo de componentes [2]. La temperatura es otro factor que influye significativamente sobre el flujo, fragilidad y consistencia del asfalto. Como el asfalto está compuesto por una mezcla compleja de hidrocarburos, la temperatura hace que la composición química cambie gradualmente, haciendo que a la vez cambien las propiedades físicas, que torna el asfalto de una consistencia semisólida a fluida. Esto, unido a las reacciones de oxidación de los componentes del asfalto cuando interaccionan con el oxígeno de la atmósfera, constituyen el fenómeno conocido como envejecimiento oxidativo de los asfaltos, el cual determina los cambios fisicoquímicos que a su vez son la base para la caracterización de los ligantes. El envejecimiento acelerado en película fina rotatoria (RTFOT), ASTM D-2872, es el método más usado para estimar cambios aproximados en las propiedades del asfalto durante la mezcla convencional en caliente a $150^{\circ} \mathrm{C}$ aproximadamente [3]. El vaso de envejecimiento a presión (PAV) está diseñado para simular el envejecimiento por oxidación a largo plazo que ocurre en los asfaltos que conforman los pavimentos. Un problema inherente en el estudio de los asfaltos es su complejidad química, que hace prácticamente imposible la separación del asfalto en componentes químicos puros. Por esta razón, los estudios químicos se han limitado a la separación de grupos genéricos, determinación de parámetros estructurales promedio e identificación estructural [4]. Algunos investigadores afirman, con base en el modelo coloidal propuesto para describir la conformación estructural del asfalto, que los cambios en la composición química resultan en una nueva estabilidad coloidal que se desvía cada vez más de las condiciones iniciales o de las condiciones óptimas de los asfaltos. El índice de Gaestel (Ic) proporciona una relación entre el envejecimiento y la estructura coloidal interna de un asfalto [5]. La espectroscopía de infrarrojo con transformada de Fourier (FTIR) ha sido ampliamente usada para identificar los grupos funcionales en el asfalto y sus fracciones. Las frecuencias características de los grupos funcionales que incluyen grupos $\mathrm{OH}$, SO y $\mathrm{NH}$ con hidrógenos libres o que forman puentes, carbonilos, ácidos carboxílicos, piridinas, pirroles, acetonas, éteres y otros, bien documentados en la literatura. En 1986, Petersen [6] propuso un método para la estimación semicuantitativa de los compuestos de bitúmenes que absorben en la región de los carbonilos. En otros estudios se ha empleado la espectroscopia FTIR para investigar las funciones de oxígeno, sulfóxido y oxígeno carboxílico en residuos de crudos de diferente procedencia. A continuación se describe en forma más detallada cada uno de los aspectos generales mencionados en esta primera parte.

\section{A. Envejecimiento oxidativo de los asfaltos}

La oxidación en los asfaltos se considera uno de los principales factores que contribuyen a su deterioro [7]. El endurecimiento y fragilización del asfalto se deben a la oxidación de ciertas moléculas del asfalto, lo cual resulta en la formación de grupos funcionales oxigenados altamente polares que interaccionan fuertemente entre sí [8]. Estudios previos han mostrado que los principales grupos funcionales resultantes de la oxidación del asfalto son los grupos carbonilo, sulfóxido y sulfonas los cuales, a su vez, son responsables del aumento en la consistencia del asfalto. El incremento en el contenido de asfaltenos se debe a la oxidación de una porción de la fase de maltenos, los cuales se tornan cada vez más polares y menos solubles en solventes orgánicos. El aumento en el contenido de carbonilos resulta de la creación de funcionalidades $\mathrm{C}=\mathrm{O}$. El contenido de carbonilos se ha usado como un indicador del grado de oxidación en los estudios sobre la cinética de oxidación en los asfaltos.

Afanasjeva y Álvarez [5] propusieron un modelo del mecanismo de envejecimiento oxidativo en los asfaltos, esquematizado en la Figura 1, con base en los resultados obtenidos en un banco de envejecimiento natural de asfaltos bajo condiciones de temperatura, humedad y radiación solar total y ultravioleta tipo A,B y C característicos del departamento de Santander, en Colombia.

FIG. 1. MECANISMO DE ENVEJECIMIENTO OXIDATIVO EN LOS ASFALTOS (AFANASJEVA Y ÁLVAREZ, 2004)

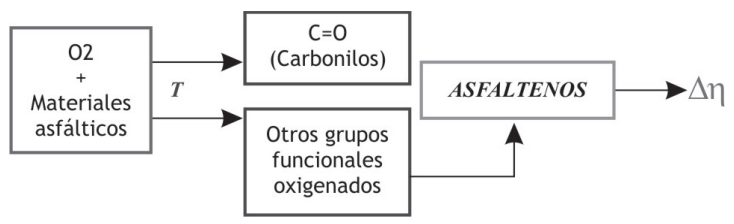

Fuente: Los autores 
En este estudio, el envejecimiento de los asfaltos fue simulado mediante ensayos de envejecimiento acelerado. Se envejecieron muestras de asfalto Apiay, Barrancabermeja y Boscán, de acuerdo a los procedimientos RTFOT y RTFTOT+PAV. Cada una de estas nueve muestras en total, fue analizada mediante fraccionamiento Corbett, espectroscopía de infrarrojo con transformada de Fourier y osmometría en fase vapor.

\section{B. Procedimiento Corbett para el fracciona- miento de los asfaltos}

Gran parte de los primeros estudios sobre asfaltos oxidados fueron realizados antes de cualquier separación, previniendo así un entendimiento completo del proceso de oxidación. Como el asfalto está compuesto de un gran número de especies, la separación total no ha sido posible. Sin embargo, se han propuesto separaciones efectivas con base en la funcionalidad. Uno de los procedimientos más usados es el desarroIlado por Corbett [9]. Esta técnica se basa en la diferencia de polaridades entre las especies químicas que constituyen el asfalto. Las separaciones según grupos o clases de compuestos sirven para dos propósitos: primero, son una herramienta analítica por sí mismas, porque proporcionan información sobre la composición de una muestra en términos de sus principales grupos y clases de compuestos. La concentración de saturados, aromáticos y polares en una muestra puede ser información suficiente. Para análisis más detallados, cada fracción puede dividirse complementariamente. Por ejemplo, los aromáticos pueden dividirse según el número de anillos aromáticos en mono-, di-, tri-, tetra- y penta-aromáticos. Sólo con esto es posible contar con valiosa información sobre la muestra sin necesidad de mediciones adicionales. De igual manera, también es ventajoso el hecho de que estas separaciones permiten una mejor caracterización molecular en mediciones subsecuentes [10]. La Figura 2 muestra esquemáticamente los pasos que sigue el procedimiento.

En esta investigación, las muestras de asfaltos iniciales y envejecidos en RTFOT y RTFOT+PAV fueron separadas en saturados, nafteno-aromáticos, polar-aromáticos y asfaltenos, según el método Corbett.
FIG. 2. ESQUEMA DE FRACCIONAMIENTO DE ASFALTOS SEGÚN EL PROCEDIMIENTO CORBETT

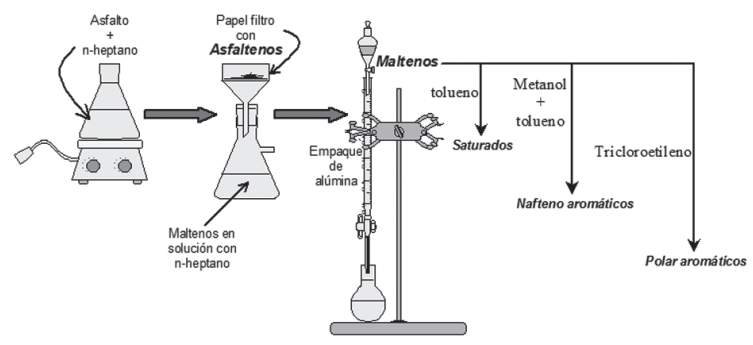

Fuente: Los autores

\section{Espectroscopía de Infrarrojo con Transforma- da de Fourier}

Muchas técnicas instrumentales, entre ellas resonancia de spin de electrón (ESR), cromatografía líquida de alta resolución (HPLC), espectrometría de infrarrojo (IR), espectrometría de masas (MS), resonancia magnética nuclear (NMR) y difracción de rayos $X$, se han empleado para caracterizar materiales asfálticos. Entre estas técnicas, la de IR es la más rápida y sensible para la detección de grupos funcionales en asfaltos, y ha probado ser una herramienta útil para el análisis de fracciones de arenas bituminosas. En la Figura 3 se muestra un espectro típico de una muestra de asfalto, que indica las asignaciones de picos según su posición.

FIG. 3. ESPECTRO INFRARROJO TÍPICO DE UNA MUESTRA DE ASFALTO

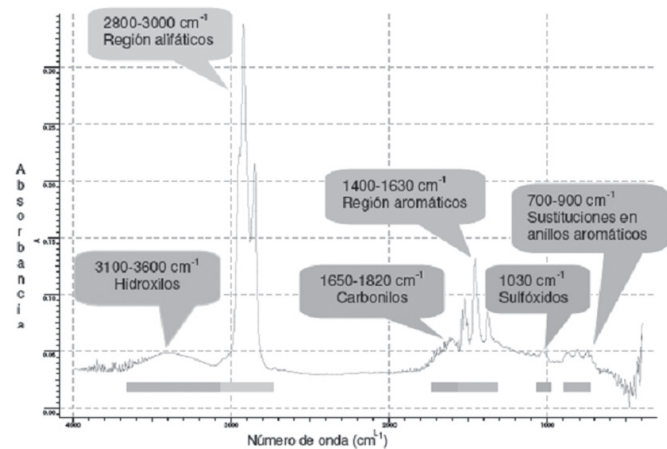

Fuente: Los autores

Los espectros se muestran como un porcentaje de transmitancia o como absorbancia versus frecuencia $\left(\mathrm{cm}^{-1}\right)$. La transmitancia $\mathrm{T}$, o porcentaje de transmitancia (100T), se define como la razón de luz transmitida sobre luz incidente, y la absorbancia $A$, es proporcional a la concentración, ra- 
zón por lo cual se usa en mediciones cuantitativas y semicuantitativas. A bajas concentraciones se cumple la ley de Beer:

$$
-\log _{10}=(T)_{v}=c \cdot l \cdot \varepsilon_{v}
$$

Donde $v$ es la longitud de onda de la luz, c es la concentración de la muestra, I es el espesor de la muestra (longitud de paso) y $\varepsilon_{v}$ es el coeficiente de absorción molecular a esa longitud de onda.

La espectroscopia IR es relativamente económica, sencilla y rápida. A pesar de que se usa ordinariamente para la identificación de compuestos simples, puede proporcionar también información rápida sobre la distribución de varios grupos estructurales y funcionales en una muestra.

Las mediciones IR pueden complementar los datos NMR. En combinación con la espectroscopia NMR, puede brindar información rápida y detallada sobre la distribución de grupos $\mathrm{CH}$. Sin embargo, en el contexto de este estudio, la principal aplicación de la espectroscopia IR es la determinación de grupos polares como $\mathrm{OH}, \mathrm{NH}$ y los diversos grupos CO [11].

\section{Aplicaciones de IR en asfaltos}

Las investigaciones adelantadas por Petersen [12] sobre puentes de hidrógeno en residuos asfálticos regulares y oxidados, mediante espectroscopia IR, aportaron significativamente al conocimiento en el campo de las fracciones pesadas del petróleo así como a la lista de asignaciones de banda y factores de respuesta.

Midiendo la absorbancia IR de estos materiales a diferentes concentraciones, Petersen demostró que a altas concentraciones los grupos $\mathrm{OH}$ y $\mathrm{NH}$, exhiben gran tendencia a formar puentes de hidrógeno. A bajas concentraciones, esa tendencia es mucho menos pronunciada. Posteriormente, Petersen [13] extendió sus investigaciones a la identificación de 2-quinolonas en los asfaltos, y sus interacciones entre ellas mismas y con ácidos carboxílicos en soluciones $\mathrm{CCl}_{4}$ así como sus interacciones en los asfaltos.

En este estudio, se obtuvieron los espectros de dos asfaltos colombianos (Apiay y Barrancabermeja) y un asfalto venezolano (Boscán) en sus estados inicial y envejecido en RTFOT y RTFOT+PAV. También se obtuvieron los espectros de las porcio- nes de maltenos y asfaltenos de cada uno de los asfaltos mencionados.

A partir de los espectros se determinaron los índices estructurales (aromaticidad, ramificación, Iongitud de cadenas) y los índices funcionales (carbonilos y sulfóxidos), de acuerdo con las siguientes expresiones [8]:

\section{Índice de aromaticidad $=$}

$$
A_{1600} / \sum A
$$

Índice de longitud de cadenas $=$

$$
A_{724} /\left(A_{1460}+A_{1376}\right)
$$

Índice de grado de ramificación =

$$
A_{2854} /\left(A_{2925}+A_{2854}\right)
$$

Índice de carbonilos $=$

$$
A_{1700} / \sum A
$$

Índice de sulfóxidos $=$

$$
A_{1032} / A_{1376}
$$

Donde $\mathrm{A}_{\mathrm{x}}$ es el valor de absorbancia a $x$ longitud de onda, y $\sum A$ está dada por la ecuación 7 :

$\sum A=A_{2925}+A_{2854}+A_{1700}+A_{1600}+A_{1460}+A_{1376}+A_{1032}+A_{724}(7)$

\section{E. Peso molecular de los asfaltos}

Varios métodos que incluyen la osmometría en fase de vapor (conocida también como osmometría de presión de vapor), ultracentrifugación, difracción y dispersión de rayos $X$, espectrometría de masas, microscopía de electrones y cromatografía de permeación en gel, han sido usados para estudiar los pesos moleculares de materiales asfálticos. Entre estos, la osmometría en fase vapor (VPO) ha sido reportada como un método conveniente para la determinación de pesos moleculares [7][14][15].

Las mediciones realizadas por osmometría en fase vapor son relativamente sencillas y económicas. Es una de las técnicas que miden propiedades coligativas, es decir, propiedades con base en la concentración del número de moléculas. 
El nombre original, osmometría de presión de vapor, sugiere que ésta mide el grado en que se disminuye la presión de vapor de un solvente en presencia de un soluto. De hecho, lo que se mide es el efecto de la temperatura, el cual está relacionado proporcionalmente con dicha reducción.

Los elementos de medición son dos termistores sensibles a la temperatura, dentro de una cámara aislada térmicamente, como se muestra en la Figura 4.

FIG. 4. ESQUEMA DE UN OSMÓMETRO DE FASE VAPOR

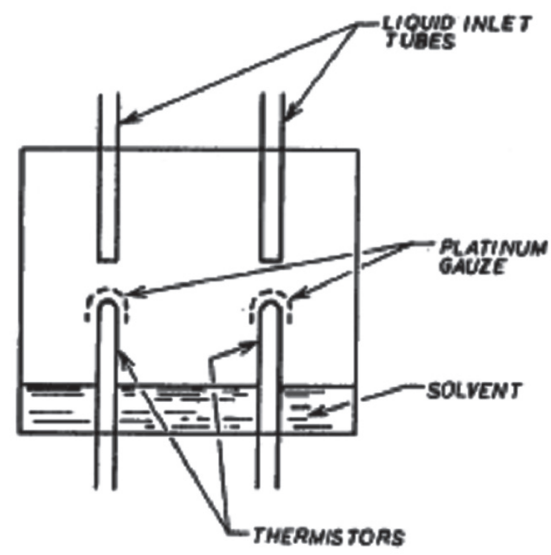

Fuente: Los autores

Usando jeringas, se deposita una pequeña gota de solvente sobre uno de los termistores, y sobre el otro se deposita una gota de solución (en el mismo solvente). Luego, la cámara se satura con vapor de solvente mientras se controla la temperatura. Como la solución tiene una presión de vapor menor que la del solvente, parte del solvente en la atmósfera de la cámara se condensará sobre el termistor. La diferencia entre las presiones de vapor es proporcional a la concentración de la muestra. El calor de condensación hace que aumente la temperatura de la gota de solución hasta que su presión de vapor sea la misma que la de los alrededores. De esta forma se establece un estado estable de condensación. El incremento en la temperatura se mide y registra. La gota de solvente sobre el otro termistor está en equilibrio con el solvente dentro de la cámara, y, teóricamente, no se produce ningún efecto térmico.

En realidad, la convección y otros efectos causan perturbaciones despreciables en el sistema. Estos efectos pueden minimizarse si se sustrae el voltaje del termistor con solvente del voltaje en el termistor con solución. La diferencia entre tempe- raturas, $\Delta T$ está relacionada con la concentración y el peso molecular (MW) a una dilución infinita mediante la Ecuación 8.

$$
\Delta T=\frac{K_{1} c}{M W}
$$

Donde $\mathrm{K}_{1}$ es una constante determinada a partir de la calibración y $c$ es la concentración de soluto. El efecto se mide a varias concentraciones. EI efecto se mide a varias concentraciones, y los resultados se grafican versus $1 / M W$ y se extrapolan a $c=0$. Los instrumentos modernos han reducido los errores experimentales a una décima del error obtenido con los primeros modelos, permitiendo la aplicación de gotas muy pequeñas de muestra sobre la punta de los termistores. Sin embargo, aún pueden ocurrir errores sistemáticos. Un problema potencial con el peso molecular promedio es que puede ser desplazado a valores menores debido a contaminantes de bajo peso molecular, como trazas de un solvente diferente del usado para la medición VPO. Por ejemplo, el tolueno residual no afectaría los pesos moleculares medidos en tolueno, pero seguramente disminuiría los MW medidos en piridina o en cualquier otro solvente. Otro tipo de problemas es el causado por efectos de agregación. Estos son los peores efectos en el caso de los componentes polares, los cuales están concentrados en las fracciones no destilables. Un solvente apropiado para VPO de hidrocarburos y compuestos ligera y moderadamente polares es el tolueno. Por ejemplo, los pesos moleculares de todos los destilados (y las fracciones no destilables solubles en pentano) concuerdan con los resultados de espectrometría de masas de ionización de campo (FIMS). Sin embargo, para los residuos altamente polares, particularmente sus fracciones no destilables, se requieren solventes más polares, como la piridina.

En este estudio, mediante osmometría de fase vapor se determinaron los pesos moleculares promedio de las muestras de asfalto Apiay, Barrancabermeja y Boscán en sus estados inicial y envejecido en RTFOT y RTFOT+PAV. También se determinaron los pesos moleculares promedio de las respectivas porciones de asfaltenos y maltenos extraídos de los asfaltos mencionados. 


\section{SECCIÓN EXPERIMENTAL}

\section{A. Objetos de estudio}

Los objetos de estudio en esta investigación fueron tres asfaltos de diferente origen. Dos de estos asfaltos son producidos en Colombia (asfaltos Apiay y Barrancabermeja, ambos con PG 64-22) y el tercer asfalto es producido en Venezuela (Asfalto Boscán, PG 70-22). Las muestras analizadas son los tres asfaltos mencionados en su estado inicial, y sus correspondientes residuos de RTFOT y RTFOT+PAV. Cada muestra fue fraccionada según el procedimiento Corbett, con el fin de estudiar la evolución de la composición genérica de los asfaltos durante el envejecimiento acelerado. Los análisis FTIR y VPO se realizaron en cada muestra de asfalto (inicial y envejecidos en RTFOT y RTFOT+PAV) y sus correspondientes porciones de maltenos y asfaltenos.

\section{B. Fraccionamiento Corbett}

Se pesaron aproximadamente 10 gramos de cada muestra de asfalto (Apiay, Barrancabermeja y Boscán, en sus respectivos estados inicial, envejecido en RTFTO y RTFOT+PAV), y se realizó la separación en saturados, nafteno-aromáticos, polar-aromáticos y asfaltenos usando el método Corbett, descrito en la norma ASTM D 4124. Esto con el fin de determinar la composición porcentual de cada asfalto y su evolución debido al envejecimiento acelerado. También se efectuaron separaciones asfalteno-maltenos a cada muestra de asfalto (3 asfaltos, iniciales y envejecidos), con el fin de hacer los análisis FTIR y VPO.

\section{Análisis FTIR}

Cada muestra de asfalto y sus respectivas fracciones de asfaltenos y maltenos fue analizada mediante espectroscopia de infrarrojo. Para esto, se disolvió cada muestra en Tetrahidrofurano, se depositó dicha solución sobre una ventana de $\mathrm{NaCl}, \mathrm{y}$ se dejó evaporar el solvente hasta que la muestra formara una película seca uniforme sobre la ventana. Los espectros infrarrojo se obtuvieron entre 4000 y $400 \mathrm{~cm}^{-1}$, con un espectrofotómetro Nicolett Nexus, con la siguiente configuración de experimento: 64 barridos, ganancia 4 y resolución 4 .

Para garantizar la significancia estadística de cada medición, se prepararon 4 ventanas con muestra, según el mismo procedimiento, y para cada ventana se hicieron 3 rotaciones, se registró un espectro por cada rotación, para un total de 12 espectros por cada muestra de asfalto en sus tres estados de envejecimiento, junto con sus respectivas fracciones de maltenos y asfaltenos.

Los espectros fueron analizados con el software con que viene equipado el espectrofotómetro, que permite definir la línea base, y calcular el área de cada pico de interés, para posteriormente evaluar los índices estructurales y funcionales mencionados en la sección 1.C.

\section{Medición de pesos moleculares mediante osmometría de fase vapor}

Para cada muestra se preparó un conjunto de soluciones en tolueno, en concentraciones ascendentes que variaron de 0,5 a $5 \mathrm{~g} / \mathrm{L}$ aproximadamente. Se prepararon soluciones de cada muestra (tres asfaltos, cada uno en estado inicial, envejecido en RTFOT y RTFOT+PAV, y sus respectivas porciones de maltenos y asfaltenos).

Se usó un osmómetro de fase vapor manufacturado por UIC Inc. Modelo 833. Antes de medir las muestras en solución, es preciso obtener la constante de calibración del osmómetro para una corriente, temperatura y solvente específicos, empleando un compuesto estándar, de peso molecular conocido. En este caso, se usó Bifenil como sustancia estándar (PM=154,21 g/mol), y el solvente para la dilución fue el tolueno anhidro. Se seleccionó una corriente de 20 A y se fijó la cámara a una temperatura de $50^{\circ} \mathrm{C}$.

Para proceder a la medición de pesos moleculares de una muestra en solución, se midió la línea base antes de cada medición. La línea base corresponde a la lectura del diferencial de voltaje $(\Delta \mathrm{V})$ para el solvente puro. Después de establecer el valor de la línea base, se procedió a registrar las lecturas de diferencial de voltaje $(\Delta \mathrm{V})$ para la solución de menor concentración (C). Se registraron varias lecturas de $\Delta \mathrm{V}$ (mínimo tres) para calcular un promedio. A este $\Delta \mathrm{V}$ promedio se le restó entonces el valor de $\Delta \mathrm{V}$ para el solvente puro (línea base), obteniéndose el valor $\Delta$ Vneto. Se siguió el mismo procedimiento con las soluciones de concentración ascendente hasta llegar a la de mayor concentración. Finalmente, se ajustan los datos 
de $\Delta$ Vneto/C vs $C$ a una línea recta y por extrapolación se determina el valor de $\Delta$ Vneto/C para una concentración cero. La constante de calibración se divide por este valor para obtener el peso molecular promedio de la muestra.

\section{RESULTADOS Y DISCUSIÓN}

\section{A. Evolución de la composición genérica con el grado de envejecimiento acelerado}

Durante la oxidación de los asfaltos tienen lugar reacciones que producen aromatización, deshidrogenación y puentes de hidrógeno ínter e intramoleculares, en grupos funcionales polares que Ilevan a la formación de más asfaltenos.

Mientras más severo sea el proceso de oxidación, mayor será el contenido de asfaltenos, que es el efecto observado en los asfaltos estudiados en esta investigación, como se observa en la Figura 5. En dicha figura se presenta la evolución en la composición fraccional de los tres asfaltos según el grado de envejecimiento acelerado. Se observa que los asfaltos Apiay y Boscán exhiben aproximadamente el mismo contenido de asfaltenos, pero a su vez, el asfalto Boscán cuenta con un contenido mucho mayor de resinas (o polar-aromáticos). Esta combinación resulta beneficiosa para el desempeño del asfalto Boscán, porque la buena dispersión de los asfaltenos depende de la cantidad de resinas y de su arreglo estructural a nivel molecular.

FIG. 5. COMPOSICIÓN GENÉRICA DE LOS ASFALTOS APIAY, BARRANCABERMEJA Y BOSCÁN SEGÚN EL GRADO DE ENVEJECIMIENTO (EN CADA CONJUNTO DE TRES FRANJAS PARA CADA FRACCIÓN, LA PRIMERA FRANJA CORRESPONDE AL ASFALTO INICIAL, LA SEGUNDA AL ASFALTO ENVEJECIDO EN RTFOT Y LA TERCERA AL ASFALTO ENVEJECIDO EN RTFOT+PAV, COMO SE INDICA SOBRE LA FRANJA DE NAFTENO-AROMÁTICOS DE APIAY, EN LA FIGURA)

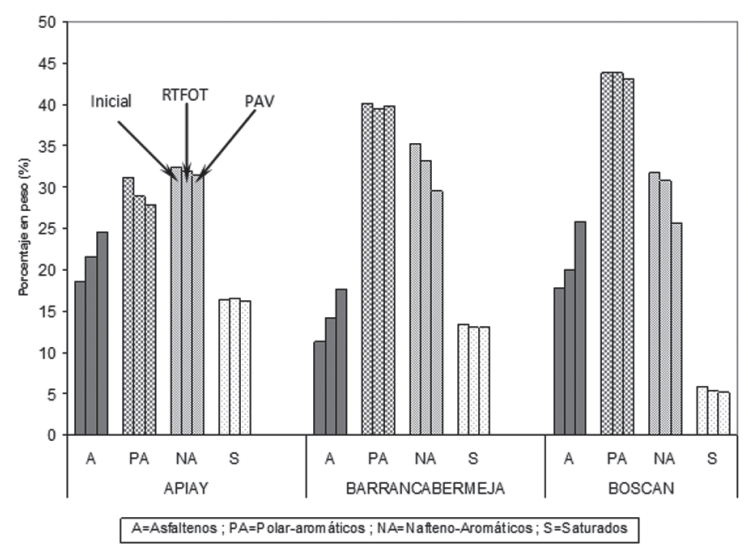

Fuente: Los autores
Otro aspecto es el bajo contenido de saturados en el asfalto Boscán. Las investigaciones han mostrado la baja compatibilidad entre asfaltenos e hidrocarburos saturados. Con base en la gráfica se puede afirmar entonces que la proporción saturados/asfaltenos del asfalto Barrancabermeja es la mayor, seguido por los asfaltos Apiay y Boscán, este último tiene una proporción saturados/ asfaltenos significativamente menor que los dos asfaltos colombianos.

La composición de los asfaltos depende considerablemente del origen del crudo a partir del cual se derivan [1]. La tendencia que exhibe cada fracción en cada asfalto sometido a envejecimientos simulados es bastante consistente con los resultados esperados. Se observa que el contenido de nafteno-aromáticos disminuye con el envejecimiento, igual que en las resinas, son estas últimas las que dan paso a la formación de asfaltenos.

Los posibles mecanismos que tienen lugar durante el envejecimiento a corto y largo plazo de estos asfaltos incluyen condensación, formación de ésteres, polimerización e isomerización, deshidrogenación, aromatización y dealquilación [13][15].

\section{B. Índice de Gaestel o de inestabilidad coloidal de los asfaltos}

Desde el punto de vista del modelo coloidal del asfalto, éste es considerado como una dispersión de asfaltenos rodeados por resinas, en un medio aceitoso [16]. Con base en esto, Gaestel definió un Índice de inestabilidad coloidal, expresado en la ecuación 9:

$$
I_{C}=\frac{\text { Asfaltenos }+ \text { Saturados }}{\text { Naftenoaromáticos }+ \text { Polar aromáticos }}
$$

El índice de Gaestel $\left(I_{c}\right)$ se usó para reflejar la relación entre el envejecimiento RTFOT y RTFOT+PAV, con la estructura coloidal de los asfaltos colombianos y el asfalto Boscán. Mientras más alto sea el índice $I_{c}$, el asfalto tenderá más a una naturaleza de tipo gel, y su estabilidad coloidal será menor [2].

La Figura 6 muestra el cambio del $I_{c}$ con el envejecimiento de los tres asfaltos estudiados. Se observa claramente que el asfalto Apiay tiene el mayor valor de este índice, mientras que los as- 
faltos Barrancabermeja y Boscán exhiben valores similares. También se observa que dicho índice aumenta a medida que el asfalto envejece, como indicador de los cambios coloidales que tienen lugar durante la transformación secuencial de nafteno-aromáticos a polar-aromáticos y asfaltenos.

\section{Análisis FTIR de los asfaltos, maltenos y as- faltenos durante el envejecimiento}

La técnica de espectroscopia de infrarrojo se empleó para estudiar cómo evoluciona la distribución de grupos funcionales presentes en los asfaltos, asfaltenos y maltenos durante el envejecimiento acelerado. Esta técnica demostró ser una herramienta muy útil para analizar cambios estructurales después de los ensayos RTFOT y PAV.

Las vibraciones más prominentes y de particular interés fueron los modos de vibración de los grupos $\mathrm{C}-\mathrm{H}, \mathrm{C}=\mathrm{O}$ y $\mathrm{S}=\mathrm{O}$. El área de cada pico se determinó mediante el método de línea base.

FIG. 6. CAMBIO DEL ÍNDICE DE INESTABILIDAD COLOIDAL O ÍNDICE DE GAESTEL $\left(I_{C}\right)$ CON EL ENVEJECIMIENTO

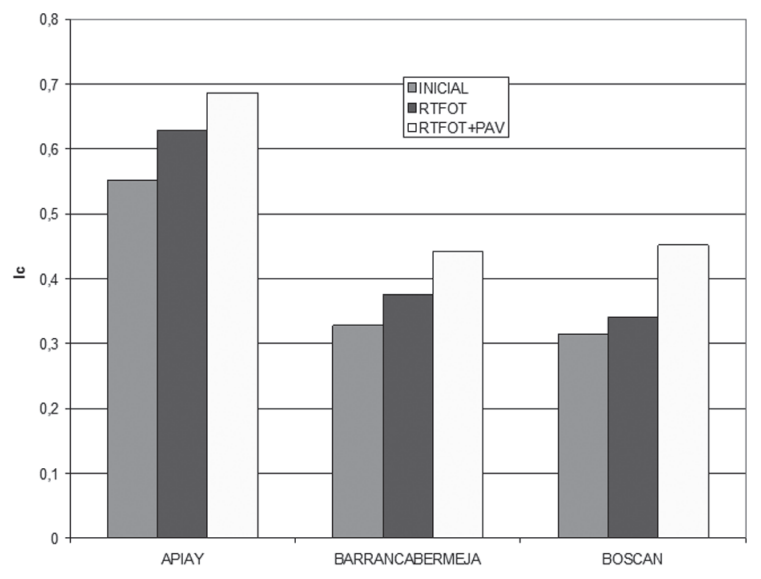

Fuente: Los autores

Los espectros exhibieron picos a 2925 y 2855 $\mathrm{cm}-1$, que indican la presencia de vibraciones de estiramiento $\mathrm{CH}$, y dos picos a 1460 y $1376 \mathrm{~cm}-1$ por las vibraciones de flexión de grupos $\mathrm{CH}$. El pico de absorción de estiramiento a $1700 \mathrm{~cm}-1$ (9) aparece debido a los grupos carbonilo $\mathrm{y} / \mathrm{o}$ carboxilo. Esta absorción cubre la región que contiene los picos de absorción de ácidos carboxílicos, cetonas y anhídridos. Las cetonas y los anhídridos se forman durante el envejecimiento oxidativo, y los ácidos carboxílicos siempre están presentes desde el estado inicial de los asfaltos, pero su contenido aumenta con el envejecimiento oxidativo. Estos tres grupos funcionales juntos (ácidos carboxílicos, cetonas y anhídridos) son las funcionalidades químicas más significativas, debido a que representan una parte integral de moléculas grandes en los asfaltos, y por lo general se les asocia con el envejecimiento oxidativo [1]. La razón principal para considerar el área total de carbonilos en vez de cada área característica es porque el espectro infrarrojo es más difícil de analizar en detalle en la región de los grupos carbonilo, debido a la aparición de la banda de las cetonas, que es muy intensa. Este pico tiene la misma frecuencia de absorción del dímero ácido, por lo que no es posible distinguir cada uno de ellos [17].

La determinación de los anhídridos formados durante el envejecimiento oxidativo tampoco es posible debido a que sus bandas de absorción quedan ocultas por la fuerte absorción de las cetonas. Los ácidos carboxílicos y las quinolonas son dos importantes grupos funcionales presentes por naturaleza en los asfaltos. Estas funcionalidades forman, entre ellas mismas y con otras especies, puentes de hidrógeno extremadamente fuertes y hacen que las bandas de absorción sean prácticamente indistinguibles [18].

Un pico intenso a $1032 \mathrm{~cm}^{-1}$ corresponde a la vibración de estiramiento de los sulfóxidos $(S=0)$, que es el grupo funcional que más fácilmente se forma durante el envejecimiento oxidativo de los sulfuros presentes en los asfaltos. En la región de $700-900 \mathrm{~cm}^{-1}$ se presentan cuatro picos fácilmente distinguibles: 870, 810, 750 y $724 \mathrm{~cm}^{-1}$ . Este último $\left(724 \mathrm{~cm}^{-1}\right)$ indica la presencia de cadenas largas de $\mathrm{CH}^{2}$.

En la Tabla 1 se presentan los parámetros calculados para los asfaltos y sus fracciones de asfaltenos y maltenos.

Los índices que exhibieron una tendencia más clara con el grado de envejecimiento son: aromaticidad, carbonilos y sulfóxidos. En el caso del índice de aromaticidad en asfaltos, éste tiende a aumentar a medida que los asfaltos envejecen. Esto concuerda con los resultados de otras investigaciones [8] y se explica con base en las reacciones de aromatización que tienen lugar en los compuestos del asfalto a medida que éste se envejece, especialmente en la porción de maltenos. 
TABLA I

PARÁMETROS FUNCIONALES Y ESTRUCTURALES DE LOS ASFALTOS, MALTENOS Y ASFALTENOS DURANTE EL ENVEJECIMENTO RTFOT Y RTFOT+PAV

\begin{tabular}{|c|c|c|c|c|c|}
\hline & \multirow{2}{*}{\multicolumn{3}{|c|}{ Índices estructurales }} & \multirow{2}{*}{\multicolumn{2}{|c|}{ Índices funcionales }} \\
\hline & & & & & \\
\hline & Aromaticidad & Ramificación & Longitud de Cadenas & Carbonilo & Sulfóxido \\
\hline \multicolumn{6}{|l|}{ ASFALTOS } \\
\hline Apiay Inicial & 0,045917 & 0,374861 & 0,01361 & 0,02872 & 0,352863 \\
\hline Apiay RTFOT & 0,049182 & 0,387135 & 0,015356 & 0,033953 & 0,461529 \\
\hline Apiay RTFOT+PAV & 0,049926 & 0,372429 & 0,013106 & 0,050917 & 1,136869 \\
\hline B/bermeja Inicial & 0,044061 & 0,351211 & 0,019042 & 0,038538 & 0,738183 \\
\hline B/bermeja RTFOT & 0,045305 & 0,356888 & 0,018741 & 0,042451 & 0,307121 \\
\hline B/bermeja RTFOT+PAV & 0,052016 & 0,382619 & 0,018214 & 0,066701 & 1,063631 \\
\hline Boscán Inicial & 0,036904 & 0,345466 & 0,019303 & 0,03548 & 0,234228 \\
\hline Boscán RTFOT & 0,039447 & 0,365517 & 0,020958 & 0,037935 & 0,406499 \\
\hline Boscán RTFOT+PAV & 0,038712 & 0,362841 & 0,02055 & 0,053787 & 1,02937 \\
\hline \multicolumn{6}{|l|}{ ASFALTENOS } \\
\hline Apiay Inicial & 0,114083 & 0,398137 & - & 0,077499 & 0,774896 \\
\hline Apiay RTFOT & 0,11399 & 0,380655 & - & 0,073876 & 0,617886 \\
\hline Apiay RTFOT+PAV & 0,111244 & 0,402863 & - & 0,124399 & 0,924041 \\
\hline B/bermeja Inicial & 0,133787 & 0,498715 & - & 0,140378 & 0,617019 \\
\hline B/bermeja RTFOT & 0,113463 & 0,408596 & - & 0,113227 & 0,555057 \\
\hline B/bermeja RTFOT+PAV & 0,118054 & 0,45757 & - & 0,174781 & 1,414354 \\
\hline Boscán Inicial & 0,113783 & 0,431151 & - & 0,082961 & 0,530816 \\
\hline Boscán RTFOT & 0,114102 & 0,454787 & 0,015673 & 0,107482 & 0,555793 \\
\hline Boscán RTFOT+PAV & 0,097708 & 0,415474 & 0,016222 & 0,132941 & 1,061812 \\
\hline \multicolumn{6}{|l|}{ MALTENOS } \\
\hline Apiay Inicial & 0,029126 & 0,367509 & 0,016569 & 0,022046 & 0,536099 \\
\hline Apiay RTFOT & 0,027113 & 0,371761 & 0,015194 & 0,025117 & 0,384462 \\
\hline Apiay RTFOT+PAV & 0,026959 & 0,374301 & 0,015785 & 0,032891 & 0,465792 \\
\hline B/bermeja Inicial & 0,029644 & 0,353769 & 0,019305 & 0,033009 & 0,177734 \\
\hline B/bermeja RTFOT & 0,029315 & 0,353772 & 0,017413 & 0,036427 & 0,285192 \\
\hline B/bermeja RTFOT+PAV & 0,029451 & 0,354415 & 0,019077 & 0,045412 & 0,821998 \\
\hline Boscán Inicial & 0,022972 & 0,347444 & 0,020432 & 0,02654 & 0,578241 \\
\hline Boscán RTFOT & 0,023343 & 0,347309 & 0,019307 & 0,028853 & 0,530027 \\
\hline Boscán RTFOT+PAV & 0,024964 & 0,344292 & 0,020766 & 0,036793 & 0,785107 \\
\hline
\end{tabular}

Fuente: Los autores

Además, el valor del índice de aromaticidad es similar para los asfaltos Apiay y Barrancabermeja, siendo el asfalto Boscán el que exhibe el menor valor, el cual está directamente asociado con el menor valor de aromaticidad de sus maltenos. La tendencia en los asfaltenos del índice de aromaticidad, de acuerdo con lo esperado, es decreciente, porque los nuevos asfaltenos formados tienen origen en las resinas, cuya proporción de aromáticos es menor en comparación con los asfaltenos presentes inicialmente en los asfaltos.
Los índices carbonilo y sulfóxido también manifiestan una clara tendencia creciente con el envejecimiento de los asfaltos. Esto se explica por la reacciones de oxidación que tienen lugar en los ligantes con el oxígeno presente durante los ensayos de envejecimiento acelerado, y concuerda con resultados reportados en la literatura [18].

Haciendo un análisis de los resultados FTIR y relacionándolos con los resultados del fracciona- 
miento Corbett para los asfaltos, se observa que los índices estructurales están relacionados en forma simple con la composición genérica de los ligantes.

\section{Peso molecular de los asfaltos, asfaltenos y maltenos}

En la Figura 7 se muestra la tendencia de los pesos moleculares de los tres asfaltos estudiados, así como sus respectivas fracciones de asfaltenos y maltenos. Se observa que el asfalto Boscán es el que menores cambios manifiesta en su peso molecular (igual que sus asfaltenos y maltenos) a medida que se somete a los ensayos de envejecimiento acelerado.

FIG. 7. INFLUENCIA DEL ENVEJECIMIENTO ACELERADO SOBRE EL PESO MOLECULAR DE ASFALTOS, ASFALTENOS Y MALTENOS

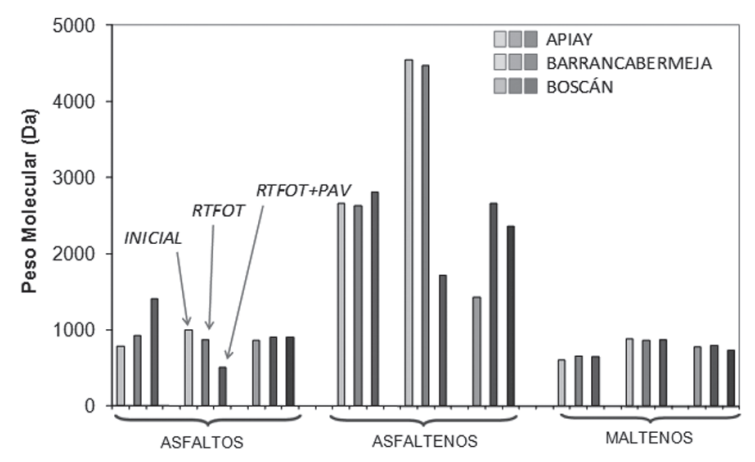

Fuente: Los autores

Nota: Cada barra corresponde a un estado de envejecimiento. La primera barra representa el peso molecular en estado inicial, la segunda barra representa la muestra envejecida en RTFOT y la tercera barra representa la muestra envejecida en RTFOT+PAV.

La tendencia de disminución de peso molecular del asfalto Barrancabermeja sugiere que las reacciones de oxidación son simultáneas con reacciones de rompimientos moleculares, que a su vez resultan en una mayor reactividad de las moléculas con el oxígeno presente en los ensayos de envejecimiento acelerado. También es evidente que el peso molecular del asfalto Barrancabermeja tiene una fuerte contribución por parte de los asfaltenos, se considera que éstos muestran la misma tendencia de disminución del peso molecular promedio que en el asfalto.
En el caso del asfalto Apiay, tanto los asfaltenos como los maltenos manifiestan cambios menores en el peso molecular a medida que el asfalto se envejece, por lo que se puede afirmar que la tendencia de aumento de peso molecular en el asfalto se debe a la asociación entre moléculas de asfaltenos y maltenos, debido a una mayor oxidación en sus componentes, lo cual resulta en mayor cantidad de puentes de hidrógeno que a su vez son responsables de la formación de mayores moléculas asociadas y mayor consistencia del ligante [10].

\section{CONCLUSIONES}

Por primera vez se llevó a cabo un análisis integral de los asfaltos colombianos y sus fracciones de asfaltenos y maltenos, en comparación con un asfalto venezolano, mediante técnicas instrumentales de alta resolución. El aporte principal de este estudio consiste en el enfoque de durabilidad de los ligantes asfálticos.

El contenido de asfaltenos así como el índice de inestabilidad coloidal Ic, aumentan a medida que los asfaltos son sometidos a diferentes niveles de severidad en ensayos de envejecimiento acelerado. No sólo el porcentaje individual de cada fracción influye sobre las propiedades del asfalto, las proporciones entre las fracciones también juegan un rol importante, debido a que algunos conjuntos de fracciones manifiestan mayor compatibilidad que otros.

El análisis FTIR de las fracciones de asfaltenos y maltenos extraídos de los asfaltos iniciales y envejecidos proporciona mayor información que el análisis FTIR de las muestras de asfalto completo, lo que permite evaluar las contribuciones de cada fracción en las características estructurales y/o funcionales de los asfaltos, al combinar los resultados de FTIR con los del fraccionamiento Corbett. Es posible obtener información aún más detallada si se analizan las fracciones de saturados, nafteno-aromáticos y polar-aromáticos por separado, lo cual permitiría evaluar la contribución de cada una de estas fracciones tanto en las características estructurales $\mathrm{y} / \mathrm{o}$ funcionales de los maltenos como en los asfaltos y cómo éstas contribuciones cambian durante el envejecimiento.

Durante el envejecimiento se forma mayor cantidad de grupos funcionales polares. Se detectó un 
aumento en los contenidos de grupos carbonilo y sulfóxido. Este incremento en compuestos oxigenados resulta en un mayor grado de asociación intra e intermolecular.

Según arreglos estructurales de las moléculas que constituyen los ligantes bituminosos, cada fracción genérica tendrá mayor o menor contribución en propiedades tales como el peso molecular promedio de los asfaltos.

\section{AGRADECIMIENTOS}

El Grupo de Investigación en Asfaltos GIAS de la Universidad Industrial de Santander agradece de manera especial al Profesor Ludo Zanzotto, Director del Bituminous Materials Chair de la Universidad de Calgary, en Canadá, y a todo su equipo de investigación, por su colaboración incondicional para permitir a la estudiante de Doctorado Juliana Puello Méndez adelantar la experimentación relacionada con esta y otras investigaciones en los laboratorios de este reconocido centro.

También agradece al Instituto Colombiano para el desarrollo de la Ciencia y la Tecnología COLCIENCIAS por el apoyo financiero para la formación de la estudiante mencionada, en el marco del Programa de Apoyo a Doctorados Nacionales.

\section{REFERENCIAS}

[1] M. Siddiqui, M. Ali, "Studies on the aging behavior of the arabian asphalts," Fuel, vol. 78, n. ${ }^{\circ}$ 9, pp. 1005 1015. 1999

[2] E. Barth, Asphalt science and technology, New York, Gordon and Breach, 1968

[3] P. Michalica, I. Kazatchov, J. Stastna, L. Zanzotto, "Relationship between chemical and rheological properties of two asphalts of different origins". Fuel, vol. 87. n. ${ }^{\circ}$ 15-16, pp. 3247-3253. 2008

[4] J.C. Petersen, R.E. Robertson, J.F. Branthaver, P.M. Harnsberger, J.J. Duvall, S.S. Kim, "Binder characterization and evaluation. Volume 4. Test methods". Transportation Research Board of the National Academies, Washington, DC, Tech. Rep. SHRP-A-370. 1994. Available: http://onlinepubs.trb.org/Onlinepubs/ shrp/SHRP-A-370.pdf

[5] N. Afanasjeva, M. Álvarez, Estudio del envejecimiento de los asfaltos colombianos bajo la acción de algunos factores climáticos,Bucaramanga, Ediciones UIS, 2004
[6] J.C. Petersen, “Quantitative differential group analysis of asphalts using differential infrared spectrometry and selective chemical reactions - Theory and applications, Prepr. 65th Annual Transportation Research Board Meeting, Washington DC, Jan 13-17. 1986

[7] J. Huang, "Oxidation of asphalt fractions," in Asphalt Science and Technology, A. Usmani, Ed., New York, Marcel Dekker, 1997

[8] J. Lamontagne, P. Dumas, V. Mouillet, J. Kister, "Comparison by Fourier transform infrared (FTIR) spectroscopy of different ageing techniques: application to road bitumens," Fuel, vol. 80, pp. 483-488. 2001

[9] L.W. Corbett, "Composition and asphalt based on generic fractionation using deasphaltening, elutionadsortion chromatography, and densimetric characterization", Analytical Chemistry, n. ${ }^{\circ} 41$, pp. 576-579. 1969

[10] K. Altgelt, M. Boduszynski, Composition and analysis of heavy petroleum fractions, New York, Marcel Dekker, 1994

[11] X. Lu, U. Isaacson, "Effect of ageing on bitumen chemistry and rheology," Construction and Building Materials, vol. 16, pp.15-22. 2002

[12] J.C. Petersen, "An infrared study of hydrogen bonding in asphalt," Fuel, n. ${ }^{\circ}$ 46, pp. 295-305. 1967

[13] J.C. Petersen, "Quantitative method using differential infrared spectrometry for the determination of compound types absorbing in the carbonyl region in asphalts," Analytical Chemistry, vol. 47 , n. ${ }^{\circ} 1$, pp. 112 117. 1975

[14] J. Huang, R. Yuro, G.A Romeo Jr.,"Photooxidation of Corbett fractions of asphalt," Petroleum Science and Technology, vol. 13, n. ${ }^{\circ}$ 9, pp. 1121-1134. 1995

[15] J.F. Branthaver, R.E. Robertson, J.J. Duvall, "Relationships between molecular weights and rheological properties of asphalts," Transportation Research Record, vol. 1535/1996, pp. 10-14. 2007.

[16] J. Speight, The chemistry and technology of petroleum, 2 ed., New York, Marcel Dekker, 1991

[17] I. Ishai, B. Brule, J.C., Vaniscote, G. Ramond, "Some rheological and physicochemical aspects of long-term asphalt durability," Proceedings of the Association on Asphalt Paving Technologists, n. ${ }^{\circ}$ 57, p.65. 1988

[18] M. Liu, M. Ferry, R. Davison, C. Glover, J. Bullin, “Oxygen uptake as correlated to carbonyl growth in aged asphalts and asphalt Corbett fractions," Industrial \& Engineering Chemistry Research, vol. 37, pp.46694674. 1998 